\section{Back and Forth}

by Renée von Paschen (C) 2012

Jumping back and forth

between languages,

I truly try

to maintain

a healthy balance.

Why does it seem

to defy

the eye,

Not to speak of

the ear?

\section{Hin und her}

German by Renée von Paschen

Beim Springen

zwischen Sprachen

Muss ich mich winden,

um den guten

Mittelweg zu finden.

Warum scheint es,

das Auge

zu täuschen,

Vom Ohr ganz zu

schweigen?

\section{Friend or Foe}

by Renée von Paschen (C) 2012

Seen from afar

you'll never know

whether friend or foe,

or only a little alliteration.

If you let go,

you'll be sure to find

there's not much of a scar

to be had from their kind.

\section{Freund oder Feind}

German by Renée von Paschen

Von weitem gesehen

fragt man sich schon,

war's Freund oder Feind

oder nur eine Alliteration.

Wer loslassen kann,

sieht mit sicherem Blick,

es bleiben kaum Narben

von beiden zurück. 


\section{Lost Language}

by Renée von Paschen (C) 2012

My lost language

has caused me much anguish

over all these years.

If it can be reinstated, without seeming too belated, I will overcome my fears.
Verlorene Sprache

German by Renée von Paschen

Meine verlorene Sprache war wie eure Rache

während all dieser Jahre.

Wenn ich sie finden kann, bevor es zu spät ist, dann werd' ich die Angst überwinden.

\section{Disdain}

by Renée von Paschen (C) 2012

You choose

to refuse

the unpleasant

truth,

As though

undoing it

would be

any use.

You never

took a

liking to

history,

Yet you have

nothing but

disdain for

her story.

\section{Verachtung \\ German by Renée von Paschen}

Du meidest

lieber

die unschöne

Wahrheit,

Als ob

leugnen

sinnvoll

wäre.

Du wolltest nicht, dass er seine

Geschichte

erzählt,

Doch ganz

verachtest du,

was für

sie zählt. 